\title{
Ein ,,anderer Liberalismus“ für das 21. Jahrhundert - aber welcher? Neue Texte von und über Judith N. Shklar
}

\author{
Rieke Trimcev (D)
}

Angenommen: 29. März 2021 / Online publiziert: 10. September 2021

(C) Der/die Autor(en) 2021

Zusammenfassung Der liberalen politischen Theorie von Judith N. Shklar wird zunehmend die Fähigkeit zugesprochen, Pfade aus der Krise der liberalen Demokratie zu weisen. Dabei lassen sich eine moderate und eine radikalere Lesart der demokratietheoretischen Anschlussfähigkeit von Shklars „Liberalismus der Furcht“ unterscheiden. Im ersten Teil des vorliegenden Aufsatzes werden diese Deutungen in der neueren Shklar-Forschung verortet; einen geeigneten Ausgangspunkt bildet die Debatte über die Frage, welche Gestaltbarkeitserwartungen Shklars skeptische politische Theorie an politisches Handeln formuliert. Zudem wird die Plausibilität der radikalen Lesart anhand der Frage diskutiert, inwiefern Shklar gewinnbringend als eine agonale Politiktheoretikerin gelesen werden kann. Im zweiten Teil werden neu edierte beziehungsweise übersetzte Texte von Shklar daraufhin befragt, welche Rückschlüsse sie auf die im ersten Teil betrachteten Debatten zulassen. Hier wird einerseits rekonstruiert, wie die Aufwertung der Demokratie im Werk von Shklar auch mit einer Aufwertung der Romantik und ihrer politiktheoretischen Ressourcen einhergeht. Andererseits wird anhand von Shklars Arbeiten über politische Verpflichtungen und zivilen Ungehorsam die behauptete Radikalität ihres Denkens abgewogen. Insgesamt plädiert der Aufsatz für die Vorzüge der moderaten demokratischen Lesart, die allerdings um einige Einsichten der radikalen Lesart erweitert werden kann.

Schlüsselwörter Judith N. Shklar · Liberalismus · Skepsis · Verpflichtung

Rieke Trimcev $(\bowtie)$

Institut für Politik- und Kommunikationswissenschaft, Universität Greifswald, Greifswald,

Deutschland

E-Mail: rieke.trimcev@uni-greifswald.de 


\title{
A Different Liberalism for the 21st Century? New Books by and on Judith N. Shklar
}

\begin{abstract}
Recent interpreters suggest that Judith N. Shklar's liberal political theory may point the way out of the crisis of liberal democracy. Situating this trend within the broader field of Shklar-scholarship, the present essay identifies and discusses a more moderate and a more radical reading of the "Liberalism of fear's" democratic resources. Tracing the topoi of this debate within newly edited or translated works by Judith Shklar, the essay argues for the advantages of the moderate reading, which can however be enriched with some of the more radical interpretation's insight.
\end{abstract}

Keywords Judith N. Shklar · Liberalism · Skepticism · Obligation

In der politikwissenschaftlichen Forschung wurden zuletzt zahlreiche Erosionsdiagnosen der liberalen Demokratie getätigt. ${ }^{1}$ So wissen wir inzwischen sehr genau, „wie Demokratien sterben“. ${ }^{2}$ Doch wissen wir auch, wie liberale Prinzipien überzeugend wiederzubeleben wären? Der an der Universität Princeton lehrende Politologe Jan-Werner Müller hat in dem vielbeachteten und mit dem Bayerischen Buchpreis ausgezeichneten Essay „Furcht und Freiheit“ vorgeschlagen, dass das Werk der in den frühen 1990er Jahren verstorbenen Politiktheoretikerin Judith N. Shklar einen, so der Untertitel, ,,anderen Liberalismus“ anzubieten habe, mit dessen Hilfe man der diskursiven und politischen Landnahme durch die Gegner der liberalen Demokratie Einhalt bieten könne. ${ }^{3}$

Während Müllers Buch eine breite öffentliche Aufmerksamkeit erfahren hat, ist eine andere, schon ein Jahr zuvor veröffentlichte Monografie über Judith N. Shklar aus der Feder der italienischen Politologin Giunia Gatta mit dem Titel „Rethinking Liberalism for the 21st Century. The Skeptical Radicalism of Judith Shklar" nur in Fachkreisen beachtet worden. ${ }^{4}$ Dabei ist es lohnenswert, beide Bücher zusammen zu lesen, weil sich so insbesondere Müllers essayistischer gehaltene Interpretation schärfer konturieren lässt. Auf den ersten Blick haben Gattas und Müllers aktualisierende Deutungen signifikante Gemeinsamkeiten: Beide dienen einem Linksliberalismus, der heute auf einem ,kleine[n], aber doch sehr umkämpften Markt für liberale meae culpae“ (Müller, S. 10) seine Verunsicherung kultiviert, Judith Shklar als star-

\footnotetext{
1 Siehe in einer früheren Ausgabe dieser Zeitschrift zusammenfassend den Rezensionsaufsatz von Höntzsch, Frauke: Krise der liberalen Demokratie? Nein, Krise des individualistischen Liberalismus, in: Neue Politische Literatur 65 (2020), H. 1, S. 255-277.

${ }^{2}$ Levitsky, Steven/Ziblatt, Daniel: Wie Demokratien sterben. Und was wir dagegen tun können, DVA, München 2018.

3 Müller, Jan-Werner: Furcht und Freiheit. Für einen anderen Liberalismus, Suhrkamp, Berlin 2019. Für eine sehr grundsätzliche Gegenposition, die sich aber tatsächlich mehr gegen Müller als gegen Shklar richtet, siehe jüngst Selk, Veith: Das Veralten des Liberalismus der Furcht, in: Martin, Susanne/Linpinsel, Thomas (Hrsg.): Angst in Kultur und Politik der Gegenwart. Beiträge zu einer Gesellschaftswissenschaft der Angst, Springer VS, Wiesbaden 2020, S. 43-60.

4 Gatta, Giunia: Rethinking Liberalism for the 21st Century. The Skeptical Radicalism of Judith Shklar, Routledge, New York, NY u.a. 2018.
} 
ke Schutzpatronin an. Mit ihrem „,negativen Egalitarismus“, der nicht Gleichheit zu verwirklichen sucht, sondern vielmehr „,die Folgen der Ungleichheit“ und die „blendende[n] Wirkung der Macht“" fürchtet, mache Shklar eine Kritik von Ungleichheitsverhältnissen aus einer genuin liberalen Perspektive möglich. Damit entkräftige die Shklar'sche Perspektive den heute politisch so effektiv mobilisierbaren Verdacht, Liberalismus sei immer ein Elitenprojekt. Sowohl Gatta als auch Müller argumentieren darüber hinaus, dass ein Shklar'scher Liberalismus für die heutige Zeit vor allem bedeute, dass sich die Ungleichheitserfahrungen von Arbeiter_innen und Migrant_innen nicht gegeneinander ausspielen lassen, sondern gemeinsam thematisiert werden können. Bei genauerem Hinsehen tun sich aber wichtige Unterschiede zwischen Müllers und Gattas Interpretation auf, die sich auf den Gegensatz zwischen einer moderaten linksliberalen Deutung und einer radikalen linksliberalen Deutung verdichten lassen.

Für Jan-Werner Müller hält „Shklars Karte“ (S. 71) zunächst eine Perspektivenverschiebung bereit. Ausgehend von der bescheiden wirkenden Frage, was vermieden werden soll, und dem Maßstab, dass systematische Furcht zu vermeiden sei, erlaube uns Shklar, alternative Positionen zu vermeintlich plausiblen politischen Gegensätzen, wie beispielsweise Populismus und Anti-Populismus, zu erschließen. Doch Shklars Lektion geht für Müller über diese Schärfung von politischer Urteilskraft hinaus. Hier liegt gerade das Innovative seiner Interpretation: Für Müller ist Shklars „Liberalismus der Furcht“ nämlich jene Spielart liberalen Denkens, die die alten Vorbehalte der Liberalen gegenüber der Demokratie hinter sich lässt und einen genuin demokratischen Liberalismus präsentiert. Damit unterstellt Müllers Buch einen Entwicklungsprozess in Shklars Denken, den er zwar selbst nicht im Einzelnen in ihren Texten rekonstruiert, der aber überzeugend scheint: Wenn aus der Sicht des „Liberalismus der Furcht“ vor allem die politische oder gesellschaftliche Konzentration von Macht gefährlich ist, dann kommt garantierten Grundrechten eine wesentliche Schutzfunktion zu. Rechte, die aber selbst niemals unumstritten sind, können nur innerhalb demokratischer institutioneller Strukturen garantiert werden: „ohne Demokratie keine Möglichkeit, auf faire Weise über die Ausgestaltung von Rechten zu streiten. Ohne Demokratie bleibt man Mächtigen ausgeliefert, die vielleicht gelegentlich gönnerhaft rechtsstaatliche Garantien gewähren, diese aber jederzeit zurücknehmen können, wenn es ihnen Vorteile verschafft [...]“ (S. 148).

Während Giunia Gatta gegen diese deutlich demokratisierte Lesart von Shklar sicherlich nichts einzuwenden hätte, will ihre eigene Interpretation doch deutlich weitergehen. In ihrer Darstellung wertet Shklar nicht nur die Bedeutung demokratischen Handelns auf, sondern wird selbst zu einer ,aktivistischen“ Denkerin. Shklar versöhne den Liberalismus nicht lediglich mit der Demokratie, sondern führe ihn zum Radikalismus der Aufklärung zurück (S. 51). Obwohl Gatta dabei immer wieder einräumt, dass Shklars ,,radikaler Liberalismus“ kein ,revolutionary and transformative design“ (S. 138) habe, klingt genau das in anderen Passagen ihres Buches immer wieder durch: Shklar gebe politischem Handeln die Würde zurück ,to bring about change, even radical change“ (S. 144). Den Unterschied zwischen „radical change“

\footnotetext{
${ }^{5}$ Shklar, Judith N.: Ganz normale Laster. Aus dem Amerikanischen übersetzt und mit einem Nachwort versehen von Hannes Bajohr, Matthes \& Seitz, Berlin 2014, S. 39.
} 
und einem „transformative design“ kann Gatta aber gerade deswegen nicht überzeugend ziehen, weil sie von Shklars Kritik an Ungleichheit auf die Aussage schließt: ,it regards the quest for freedom and equality as always unfinished and incomplete“ (S. 144). Dass Shklar zwischen einem ,quest for equality“ (auch einem immer unvollkommenen) und einer ,avoidance of inequality“ einen zentralen Unterschied machen würde, sieht Gatta nicht.

Die Kontroverse, die sich aus dieser knappen Gegenüberstellung von Müller und Gatta ergibt, lässt sich in zwei Debattensträngen der Sekundärliteratur spiegeln und weiter aufschlüsseln. Dies wird mir im ersten Teil dieses Rezensionsessays erlauben, auch die Beiträge der Anthologie „Between Utopia and Realism. The Political Thought of Judith N. Shklar“ zu würdigen, den Samantha Ashenden und Andreas Hess herausgegeben haben; ${ }^{6}$ auch werde ich auf einige neuere Zeitschriftenbeiträge über Shklar eingehen. Ich blicke in diesem ersten Teil zunächst auf Shklars bereits vieldiskutierte skeptische Art, politische Theorie zu betreiben; fraglich ist dabei, welchen Anspruch eine solch skeptische politische Theorie an politische Gestaltbarkeit stellt und stellen kann. Dies ermöglicht es mir, zunächst diejenigen Aspekte zu hinterfragen, die die Deutungen von Gatta und Müller miteinander teilen. Denn hier sollen insbesondere auch solche Positionen Beachtung finden, die die Porträtierung des „Liberalismus der Furcht“ als vielversprechende Alternative mit einem Fragezeichen versehen. Anschließend richte ich mein Augenmerk auf die Differenzen zwischen Gattas radikaler und Müllers moderater Deutung von Shklar, indem ich Gattas Charakterisierung von Shklars Ansatz als einer ,agonalen“ politischen Theorie eingehend diskutiere.

Im zweiten Teil dieses Besprechungsessays wende ich mich dann neuen, das heißt erstmals aus dem Nachlass edierten beziehungsweise erstmals ins Deutsche übersetzten Texten von Shklar zu, nämlich „Über Hannah Arendt, Verpflichtung, Loyalität, Exil“ und „On Political Obligation“.7 Es wird gefragt, welches Licht sie auf die im ersten Teil skizzierten Kontroversen werfen und welche Urteile der Interpreten revisionsbedürftig erscheinen. Wichtig ist darüber hinaus, welche neuen Fragen sie auf die Agenda der Shklar-Forschung setzen. ${ }^{8}$

\section{Neue Literatur über Judith Shklar}

\subsection{Liberale Skepsis und politische Gestaltbarkeit}

Shklar bezeichnete ihre eigene Haltung als politische Theoretikerin immer als eine skeptische. Bereits die aus ihrer Dissertation hervorgegangene Monografie „After

\footnotetext{
6 Ashenden, Samantha/Hess, Andreas (Hrsg.): Between Utopia and Realism, Pennsylvania UP, Philadelphia, PA 2019.

7 Shklar, Judith N.: Über Hannah Arendt. Herausgegeben und mit einem Nachwort versehen von Hannes Bajohr, Matthes \& Seitz, Berlin 2020; Shklar, Judith N.: Verpflichtung, Loyalität, Exil. Mit einem Essay über Michael Walzer, Herausgegeben und übersetzt von Hannes Bajohr, Matthes \& Seitz, Berlin 2019; Shklar, Judith N.: On Political Obligation. Edited and with an Introduction by Samantha Ashenden and Andreas Hess, Yale UP, New Haven, CT u. a. 2019.

8 An dieser Stelle möchte ich Milos Rodatos für seine hilfreichen Anmerkungen zu diesem Text danken.
} 
Utopia. The Decline of Political Faith“ beendete sie mit dem Urteil: „A reasoned skepticism is [...] the sanest attitude for the present" ${ }^{\circ}{ }^{9}$ Die Erfahrung des Totalitarismus habe fortschrittsoptimistische Positionen diskreditiert. Zugleich hält Shklar aber auch fatalistische und kulturpessimistische Deutungsmuster für grundsätzlich ungeeignet, die Konsequenzen und Erfahrungen des Totalitarismus zu erschließen. Shklars selbsternannte Skepsis ist in der Vergangenheit der Ausgangspunkt verschiedenster Interpretationen geworden. ${ }^{10}$ Mit den hier besprochenen neueren Beiträgen geht diese besonders fortgeschrittene Debatte in der Shklar-Forschung in eine neue Runde. Dabei zeichnet die neuen Beiträge aus, dass sie die Frage nach der genauen Natur von Shklars Skepsis, nach ihrem Verhältnis zur normativen Reichweite des Shklar'schen Liberalismus, sowie schließlich nach ihrer politischen Stoßrichtung mit einem geschärften Blick für Entwicklungen und Diskontinuitäten im Schaffen der amerikanischen Politiktheoretikerin verbinden.

Ausgehend von Giunia Gattas Interpretation lassen sich die unterschiedlichen vertretenen Positionen gut einander gegenüberstellen. Für Gatta ist Shklars Skepsis das zentrale und verbindende Merkmal ihres politischen Denkens (S. 7, 36). Dabei sieht die italienische Politologin die Shklar'sche Skepsis in einem doppelten Verwandtschaftsverhältnis. Erstens sei das, was Shklar in „After Utopia“ einen ,reasoned skepticism“ nannte, eine Anverwandlung des ,,age of skepticism“ - der Aufklärung. Indem Shklar in „After Utopia“ die romantische und christliche Kritik an der Aufklärung kritisiere, bringe sie eine Hoffnung auf eine Rückkehr zum Optimismus der Aufklärung zum Ausdruck (S. 61). Zweitens liest Gatta - wie auch schon vor ihr Stephen White und Kamila Stullerova ${ }^{11}$ - Shklar als liberale Stimme einer ,nonfoundational political theory“, die schon in den 1950ern postmoderne topoi der Modernekritik vorweggenommen habe (S. 3, 47). In dieser Familienaufstellung ist es nicht weiter verwunderlich, dass Shklars Skepsis für Giunia Gatta immer zugleich radikal, und das heißt bei ihr ,aktivistisch“, ,progressiv“ oder ,transformativ“ ist (S. 3 und 11) - und dieser „skeptische Radikalismus“, so die Hauptbotschaft des Buches, entfalte sich langsam aber stetig in Shklars Werk.

Auch Samuel Moyn liest „After Utopia“ ganz wie Gatta als „longing for a lost radicalism“ (Gatta, S. 61), als Suche nach einer Alternative zum konservativ werdenden „Cold War Liberalism“. Dabei besticht sein Aufsatz in „Between Utopia and Realism“ durch eine esoterische Verteidigung dieser Lesart. ${ }^{12}$ Denn Moyn gibt den kontraintuitiven Charakter dieser Interpretation unumwunden zu. Wohl kein Satz aus „After Utopia“ wurde so häufig zitiert wie Shklars einleitende Standortbestimmung: „The author shares in the spirit of the age to the extent of being neither able nor willing to build an original theory of politics. The fact is that it is next

\footnotetext{
${ }^{9}$ Shklar, Judith N.: After Utopia. The Decline of Political Faith, Princeton UP, Princeton, NJ 1969, S. 272f.

${ }^{10}$ Siehe zum Beispiel Forrester, Katrina: Hope and Memory in the Thought of Judith Shklar, in: Modern Intellectual History 8 (2011), H. 3, S. 591-620; Stullerova, Kamila: Embracing Ontological Doubt: The Role of ,Reality“ in Political Realism, in: Journal of International Political Theory 13 (2017), H. 1, S. 59-80.

11 White, Stephen K.: Political Theory and Postmodernism, Cambridge UP, Cambridge 1991, S. 123; Stullerova: Doubt (wie Anm. 10).

12 Moyn, Samuel: Before - and Beyond - the Liberalism of Fear, in: Ashenden: Utopia (wie Anm. 6), S. 24-46.
} 
to impossible to believe strongly that the power of human reason expressing itself in political action is capable of achieving its ends". ${ }^{13}$ Moyn gibt nun zu bedenken: Gerade dass Shklar in so deutlicher Manier analysiere, warum der Optimismus der Aufklärung unglaubwürdig geworden sei, ließe die Situation der politischen Theorie als unhaltbar erscheinen. Ihre Kritik, ,put the theoretical premium on the conditions for a believable Enlightenment and an actionable radicalism. That Shklar personally did not develop them, rallying soon after to ,survivalism" among the ruins, changes nothing“ (S. 42). Dennoch biete „After Utopia“, getrennt von Shklars restlichem Werk, allen, die den konservativen Gestus ablegen und sich zum Erbe der radikalen Aufklärung zurückwagen wollen, einen geeigneten Ausgangspunkt. Obwohl Moyn und Gatta Shklars Erstling damit durchaus ähnlich lesen, könnte ihre Perspektive auf das, was werkbiografisch auf „After Utopia“ folgt, kaum unterschiedlicher sein. Während sich der implizite Radikalismus von „After Utopia“ für Gatta werkbiografisch zunehmend Geltung verschafft, kommt es für Moyn in dieser Hinsicht zu einer Zäsur: Ab „Legalism“, Shklars zweiter Monografie aus dem Jahr 1964, ergebe sich die Politiktheoretikerin zunehmend einem konservativen Liberalismus.

Doch in welchem Maße schlägt sich Shklars selbsterklärte Skepsis in „After Utopia“ tatsächlich auf die Seite des Radikalismus der Aufklärung? Wie viel Hoffnung nach einer optimistischen Theorieoption steckt in ihrem Buch? Als interessant könnte sich hier ein Vergleich zwischen „After Utopia“ und dem Text, aus dem das Buch entstand, nämlich Shklars Dissertation „Fate and Futility. Two Themes in Contemporary Political Theory“ erweisen. Tatsächlich expliziert Shklar auf den ersten Seiten dieses Texts ihr eigenes Politikverständnis wie folgt:

„To begin with, then, the idea of any laws of history, of any sort of inevitability is absolutely rejected, and every historical generalization regarded with great scepticism. Political activity, especially, seems to be free from determinants external to its sphere. The pursuit and exercise of power, should by definition imply a conscious and purposeful imposition of their will by individuals and groups upon the existing order of society“. ${ }^{14}$

Im Vergleich zu ihrer Charakterisierung des radikalen Politikverständnisses der Aufklärung fällt auf, dass Shklar hier zwar den aufklärerischen Optimismus zurückweist; an der Prämisse politischer Gestaltbarkeit ${ }^{15}$ möchte sie allerdings offensichtlich festhalten. Wenn Shklar also in den 1950er Jahren tatsächlich die von Gatta und Moyn wahrgenommene Hoffnung hegte - hier wäre sie explizit verbürgt. Allerdings lohnt sich ein Blick auf das Schicksal dieser Passage. Denn sie schaffte es nie in die überarbeitete und zwei Jahre später veröffentlichte Fassung von Shklars Dissertation. Vielmehr nahm Shklar hier einiges an Verve zurück: „The question is whether politics, the ability to act freely in history, is still able to offer a means of

\footnotetext{
13 Shklar: After Utopia (wie Anm. 9), S. ix.

14 Shklar, Judith N.: Fate and Futility. Two Themes in Contemporary Political Theory, Cambridge, MA 1955, S. 2. Mit freundlicher Genehmigung der Harvard University Archives.

15 Shklar nennt diesen Glauben an ,,men [...] as the free creators of society“ in „After Utopia“ den ,Anarchismus“ der Aufklärung, Shklar: After Utopia (wie Anm. 9), S. 5.
} 
social improvement ". ${ }^{16}$ Dies ist ein Indiz dafür, dass sie keine geheime Brücke zum skeptischen Radikalismus der Aufklärung schlägt.

Auf der Suche nach weiteren Kontrapunkten zu Gattas und Müllers Vertrauen auf den „Liberalismus der Furcht“ lohnt ein Blick in einen jüngeren Zeitschriftenbeitrag des irischen politischen Philosophen Allyn Fives. Wie Moyn möchte auch Fives die frühe gegen die späte Shklar starkmachen. Allerdings gerade nicht, weil Shklars späterer „Liberalismus der Furcht“ ihm zu konservativ und minimalistisch erscheint - im Gegenteil, für Fives ist der „Liberalismus der Furcht“ gerade zu „,maximalistisch" in seiner epistemologischen und wohl auch normativen Ambition. ${ }^{17}$ Anders als Moyn sieht Fives die entscheidende Kehrtwende in Shklars Werk auch nicht zwischen dem Erstling „After Utopia“ und „Legalism“, sondern zwischen „Legalism“ und Shklars folgender Schaffensphase unter dem Zeichen des vielzitierten „Liberalismus der Furcht“. Shklar bleibe zwar durchgehend Skeptikerin, was für Fives zunächst Skepsis an der ideellen Theorie ihrer Zeitgenoss_innen und eine Parteinahme für eine realistische politische Theorie bedeutet. In „Legalism“ kombiniere Shklar diese Skepsis mit einem Werte-Pluralismus, der von der Existenz von ,incompatible moral claims“ und der Abwesenheit einer ,general rule for their resolution“ ausgehe. ${ }^{18}$ Mit dem „Liberalismus der Furcht“, der Grausamkeit als summum malum an die erste Stelle der zu vermeidenden Übel erklärt, gehe Shklar allerdings zu einer monistischen Position über. Denn Shklar erhebe nun den Anspruch, mit der Vermeidung von Grausamkeit ein allgemeines Prinzip zur Entscheidung von moralischen Konflikten gefunden zu haben. Autor_innen wie Kamila Stullerova, Philip Spencer oder Giunia Gatta sehen diese ,unique blend of skepticism and commitment“ (Gatta, S. 2) als Stärke von Shklars mittlerer Werkphase; so argumentiert beispielsweise Stullerova in „Between Utopia and Realism“, dass Shklars gleichermaßen realistisches wie kosmopolitisches Prinzip ,putting cruelty first“ eine differenzierte Bewertung von humanitären Interventionen erlaube. ${ }^{19}$ Fives dagegen hält eben jene Verbindung von Skepsis und einer deutlichen normativen Positionierung im Falle von Shklar für inkohärent, und wirbt für die Vorzüge des skeptischen Pluralismus der frühen Judith Shklar.

Dass Shklar-Interpret_innen zunehmend versuchen, der Versuchung der Konstruktion eines „Mythos der Kohärenz“20 zu widerstehen, kann als Zeichen eines

\footnotetext{
16 Ebd., S. $216 f$.

17 Fives, Allyn: The Unnoticed Monism of Judith Shklar's Liberalism of Fear, in: Philosophy \& Social Criticism 46 (2020), H. 1, S. 45-63. Zwischen der Einreichung und der Veröffentlichung des vorliegenden Beitrags hat Fives seine Shklar-Interpretation in Buchlänge veröffentlicht, siehe Fives, Allyn: Judith Shklar and the liberalism of fear, Manchester UP, Manchester 2020. Die Monografie konnte in dieser Besprechung leider nicht mehr berücksichtigt werden.
}

18 Fives: Monism (wie Anm. 17), S. 47.

19 Stullerova, Kamila: Cruelty and International Relations, in: Ashenden: Utopia (wie Anm. 6), S. 67-85. Eine ähnliche Bedeutung von Shklars Ansatz für die Einschätzung von Verbrechen gegen die Menschlichkeit siehe Spencer, Philip: „Putting Cruelty First‘: The Summum Malum, Genocide, and Crimes Against Humanity, in: Ashenden: Utopia (wie Anm. 6), S. 198-218.

20 Zum hermeneutischen Laster der Konstruktion von Kohärenzmythen siehe Skinner, Quentin: Bedeutung und Verstehen in der Ideengeschichte, in: Ders.: Visionen des Politischen, Suhrkamp, Frankfurt a. M. 2009, S. 21-63, hier S. 35-41. 
erfreulichen Fortschritts gewertet werden. Gegenüber den starken und gegensätzlichen Diskontinuitätsthesen von Moyn und Fives ist allerdings die Frage anzubringen, wie sie sich zu Shklars Spätwerk verhalten. Bleibt Shklars Liberalismus wirklich so minimalistisch, wie Moyn es behauptet? Und ist er tatsächlich so sicher in seiner epistemischen Grundierung und so zuversichtlich in seiner normativen Reichweite seines Grausamkeitsvermeidungsgebotes, wie es Fives mit seiner Monismus-These unterstellt? Es ist in diesem Zusammenhang das Verdienst der Interpretation von Hannes Bajohr, dass er mit einer differenzierten Entwicklungsthese ein Deutungsangebot für die von Müller angenommene Evolution in Shklars Werk anbietet; dabei vermag er zugleich den Eindruck von Fives, dass Shklars Liberalismus ab den 1980er Jahren an normativem Gehalt gewinnt, gewinnbringend auszubuchstabieren. ${ }^{21} \mathrm{Ba}-$ johrs Deutung setzt sich in zwei Punkten von derjenigen Fives' ab: Erstens bestimmt er das Verhältnis zwischen Shklars Skepsis und ihrer Forderung, Grausamkeit an erste Stelle zu setzen, anders. Für Fives begründet das Grausamkeitsvermeidungsverbot Shklars Skepsis: „It is because human cruelty is so pervasive that we are forced to doubt what we know“. ${ }^{22}$ Für Bajohr ist die in „Ganz normale Laster“ formulierte Forderung, Grausamkeit allen anderen Lastern voranzustellen, ein Ausdruck einer skeptischen Haltung. Bajohr kann zu einer unterschiedlichen Einschätzung gelangen, weil er Shklars Skepsis anders bestimmt als Fives. Bajohr betont, dass Shklars Skepsis nicht vordergründig eine epistemische Skepsis ist, die an den Möglichkeiten von Wissen zweifelt, sondern eine politische Skepsis, die kritisch auf gesellschaftliche Konventionen und ihre politischen Machteffekte schaut. Aufbauend auf dieser Differenzierung kann Bajohr die Normativität von Shklars Liberalismus auf eine Weise rekonstruieren, die eine Alternative zu der monistischen Lesart von Fives aufzeigt. Bajohr unterscheidet gleich drei „Quellen liberaler Normativität“ bei Shklar: Zunächst baue Shklar auf das empirische Prinzip der Furcht vor Grausamkeit. Dieses werde aber zunehmend durch ein formales Prinzip überschrieben, nämlich durch eine reflexive „Furcht vor der Furcht“ (S. 170). Dieses Prinzip erlaube Shklar einen kontextsensiblen Universalismus, der pluralen Quellen von Furcht gerecht werden könne und diese auch zunehmend extensiv auslege. So erinnert Bajohr daran, dass Shklar in „American Citizenship“ so weit gehe, aus der Furcht vor Arbeitslosigkeit ein Recht auf Erwerbsarbeit abzuleiten. ${ }^{23}$ Auf diese Weise eröffnet der „Liberalismus der Furcht" einen nicht unbeträchtlichen Raum für konfligierende moral claims, und es wird mehr als fraglich, ob er selbst die Ressourcen besitzt, eine eindeutige Urteilsregel für ihre Abwägung bereitzustellen, so wie Fives es mit seiner MonismusThese annimmt. Für die Frage nach einer demokratischen Wende des „Liberalismus der Furcht" wird dann bei Bajohr eine dritte, transzendentale Quelle von Normativität relevant. Diese zielt auf die Bedingungen der Möglichkeit, ein Objekt der Furcht

\footnotetext{
21 Bajohr, Hannes: Judith N. Shklar's Sources of Normativity, in: Ashenden: Utopia (wie Anm. 6). Dieser Aufsatz ist auch auf Deutsch erschienen: ders.: Judith N. Shklar über die Quellen liberaler Normativität, in: Fischer, Karsten/Huhnholz, Sebastian (Hrsg.): Liberalismus. Traditionsbestände und Gegenwartskontroversen, Nomos, Baden-Baden 2019, S. 71-98. Ich zitiere in der Folge aus der englischen Fassung.

22 Fives: Monism (wie Anm. 17), S. 52.

23 Ebd., vergleiche auch Shklar, Judith N.: American Citizenship. The Quest for Inclusion, Harvard UP, Cambridge/MA u.a. 1991.
} 
auch als ein solches gesellschaftlich artikulieren zu können und so seinen „Sinn für Ungerechtigkeit“" zum Ausdruck zu bringen (S. 171).

Mit dieser Interpretation von Hannes Bajohr schließt sich zugleich der Kreis zu der eingangs referierten Position von Gatta. Denn während auch Bajohr mit seiner dritten Quelle liberaler Normativität einen konstitutiven Bezug zu demokratischen Prinzipien freilegt, rekonstruiert er dies als eine späte Entwicklung in Shklars Denken, und nicht als einen bereits in den 1950er Jahren angelegten und sich zunehmend entfaltenden Kern ihres Liberalismus.

\subsection{Ein agonaler Liberalismus?}

Dass Shklars Liberalismus politische Konflikte aufwertet, ist in der Sekundärliteratur zuletzt mehrfach betont worden. ${ }^{24}$ Auch Müllers Essay läuft auf von Shklar inspirierte Empfehlungen hinaus, wie es sich auf zeitgenössischen politischen Kampfplätzen mehr und besser streiten ließe. Die Monografie von Giunia Gatta unterbreitet hier ein willkommenes Systematisierungsangebot, indem sie Shklar als ,,agonale“ Politiktheoretikerin deutet. Autor_innen wie Chantal Mouffe, William Connolly, James Tully oder Bonnie Honig haben den agon in den letzten Jahren als verheißungsvolles Modewort etabliert, dessen Gebrauch aufmerksamkeitsökonomische Vorteile verspricht. Für die Shklar-Interpretation von Giunia Gatta leistet diese beliebte Semantik allerdings noch mehr: Der Einordnung als ,agonaler" Ansatz kommt eine wichtige argumentative Beweislast für den Nachweis der Radikalität von Shklars Denken nach. Insofern lässt sich hier exemplarisch fragen, inwiefern Gattas radikale Lesart gegenüber Müllers moderater Lesart überzeugen kann.

Systematisiert man Gattas Gebrauch des Begriffs ,agonal', so lassen sich vier Eigenschaften identifizieren, die mit diesem Attribut an Shklars Perspektive auf politische Konflikte hervorgehoben werden: Erstens sei Shklars eigenes politiktheoretisches Selbstverständnis agonal, weil sie ihr eigenes Schreiben als ,,a firmly asserted, reasoned intervention in the field of politics, held with great conviction but not grounded as true on nature, the order of things, or even history“ (S. 110) verstehe. Zweitens vertrete Shklar einen Liberalismus, der pluralistische Konflikte nicht nur in einen Zustand der Toleranz co-existierender Werte und politischer Ansichten überführen wolle, sondern auch danach frage, was denn das Gemeinsame darstelle, was einen Konflikt als agon auszeichne (also aus Feinden Gegner zu machen, um in den Worten von Chantal Mouffe zu sprechen), und so den politischen Disput erst lebendig werden lasse (S. 117). Gatta zufolge ist das die Furcht, die gerade nicht als normatives Fundament von Shklars Liberalismus zu gelten habe, sondern als geeigneter Ausgangspunkt, ,to open an agon about what fear is, or rather what it means in different settings, situations, and cultural contexts" (S. 115). Drittens kann Shklars Theorie als ,agonal' aufgrund der Annahme bezeichnet werden, dass

\footnotetext{
24 Bülte, Tobias: Politisches Denken jenseits von Begründung und Fatalismus. Überlegungen zu einer erweiterten Lesart Judith Shklars, in: Zeitschrift für Politische Theorie 9 (2018), H. 2, S. 193-208; Trimçev, Rieke: Verbindlichkeitskonflikte und politische Verpflichtung, in: Zeitschrift für Politische Theorie 9 (2018), H. 2, S. 253-267; Kaufmann, Katharina: Conflict in Political Liberalism. Judith Shklar's Liberalism of Fear, in: Res Publica 26 (2020), H. 4, S. 577-595.
} 
die politische Konfliktaustragung immer in einem Kontext asymmetrischer Machtverteilung stattfindet (S. 117). Es ist eine Stärke von Gattas Monografie, Shklars Sensibilität für Ungleichheit besonders herauszuarbeiten. Gerade ihre hervorragende Rekonstruktion der Bedeutung von Jean-Jacques Rousseaus ,everyman“ für Shklars Werk (S. 65-88) lässt das Urteil von Volker Heins in „Between Utopia and Realism“ verstehen, demzufolge Shklar der Kritischen Theorie in Kritikfähigkeit in nichts nachstehe. ${ }^{25}$ Viertens schließlich identifiziert Gatta auch eine weitere für agonale Theoretiker_innen typische Annahme: So habe sich Shklar seit „Legalism“ stets für die Rückwirkungen und Interaktionen zwischen politischem Handeln und institutionellem und rechtlichem Regelwerk interessiert (Gatta, S. 111). Alle vier Elemente sind in der Tat wichtige Merkmale der sogenannten agonalen Demokratieoder Politiktheorie.

Überraschend ist, dass Gatta kaum den expliziten Bezug zu dieser zeitgenössischen Literatur sucht, die auch im angloamerikanischen Raum schon eine eigentlich nicht zu übersehende Debatte in Fachjournalen nach sich gezogen hat. Mit einem Seitenblick auf Nietzsche und Arendt, die nun gerade nicht als natürliche Verbündete von Shklar gelten können, entlehnt Gatta den Ausdruck ,agonistic liberalism“ der Berlin-Interpretation von John Gray. ${ }^{26}$ Dabei will sie die von Gray vorgeschlagene Begriffsverwendung aber gerade nicht übernehmen, sondern im Grunde Shklars radikaldemokratische Anknüpfungspunkte hervorkehren. Insofern verwundert es umso mehr, dass der Vergleich mit, ja sogar jeglicher Verweis auf Autor_innen wie Mouffe oder Honig ausbleibt.

Ohne diesen Vergleich hier nachholen zu können, seien hier doch wenigstens einige Zweifel daran angezeigt, wie viele Gemeinsamkeiten er zu Tage fördern würde. Schon Hannes Bajohr hat gute Gründe dafür angeführt, dass Shklar die postfundamentalistischen Prämissen der meisten der oben genannten agonists nicht teilt: Erstens fiel die für diese Richtung konstitutive epistemische Skepsis, also der Zweifel an der Möglichkeit von Wissen, bei Shklar sehr viel weniger grundsätzlich aus (Bajohr in Ashenden: Utopia, S. 163); zweitens war es nicht die epistemische Skepsis, sondern eine politische Skepsis, das heißt eine zweifelnde Haltung gegenüber allen sozialen Konventionen, die für ihre Parteinahme für den Liberalismus ausschlaggebend war (S. 162). Zudem kann ich nicht erkennen, dass Shklar ihre Perspektive auf Ungleichheit und Machtasymmetrie auf eine Unterscheidung zwischen „Politik“ und „,dem Politischen“ aufbauen würde, die für radikaldemokratische agonists so typisch ist.

Wenn aber Gattas Intuition, dass Shklars Ansatz gewinnbringend als ein agonaler zu verstehen wäre, aufgenommen werden soll, könnte es sich als ertragreicher erweisen, Shklars Alleinstellungsmerkmale als liberale agonale Theoretikerin zu betonen. Gattas Intuition, dass Shklars ,,agonistic liberalism“ stärker wäre als der Gray-Berlin'sche, scheint dabei durchaus in eine richtige Richtung zu weisen. Auch hier hätte man sich einen noch eingehenderen Vergleich gewünscht. So wäre es

\footnotetext{
25 Heins, Volker M.: ,More modest and more political‘. From the Frankfurt School to the Liberalism of Fear, in: Ashenden: Utopia (wie Anm. 6), S. 179-197, hier S. 181.

26 Gray, John: Isaiah Berlin. An Interpretation of His Thought, Princeton UP, Princeton, NJ 2020, S. 175-202.
} 
spannend gewesen mit Shklar gegen Gray zu zeigen, dass ein „,agonistic liberalism“, der den meisten der Kriterien von Gray entspricht, zugleich ohne die „ineliminable communitarian dimension" und den konstitutiven Bezug auf die Nation auskommt, die Gray für notwendig hältt. ${ }^{27}$ Meines Erachtens schießt Gatta über das Ziel hinaus, wenn sie die Abgrenzung dazu nutzt, Shklars ,,agonistic liberalism“ im Zuge ihrer post-fundamentalistischen Interpretationslinie, trotz des liberalen Labels in der Sache, in einem radikaldemokratischen Projekt aufgehen zu lassen.

Spannend wäre es in dieser Hinsicht, den Blick auf die Frage nach der institutionellen Dimension von Shklars Denken zu lenken. Wenn ein agonaler Liberalismus gegenüber anderen Modellen einen Vorzug haben sollte, dann wäre es ein fruchtbarerer und offenerer Blick auf die Frage nach politischen Institutionen, für deren Ausblenden radikaldemokratische Ansätze zuletzt viel gescholten wurden. Dass Shklars Denken institutionentheoretisch anschlussfähig ist, wurde in der Sekundärliteratur immer wieder betont. ${ }^{28}$ Allerdings ist eine ausführlichere Auseinandersetzung bisher ausgeblieben. Diese Lücke bearbeitet ein Beitrag von William E. Scheuerman, und zwar mit durchaus kritischer Pointe. ${ }^{29}$ Scheuerman bemerkt zunächst, dass Shklars „Liberalismus der Furcht“ der 1980er Jahre ein ambivalentes Verhältnis zu rechtstaatlichen Institutionen unterhält. Auf der einen Seite suggeriere Shklar, dass der „Liberalismus der Furcht“ eine eigenständige Rechtfertigung von Rechtstaatlichkeit zulasse, da dieser im Schutz gegen Willkür und Grausamkeit eine zentrale Rolle zukomme. Auf der anderen Seite, so gibt Scheuerman aufmerksam zu bedenken, schlichen sich über Shklars Rezeption von Michel de Montaigne gewisse anti-legalistische Argumentationsfiguren in ihr Denken, denen zufolge allgemeine Regeln immer Gefahr liefen, individuelle Erfahrungen auszublenden. Mit dieser ihm eigenen Arroganz würde der Rechtsstaat gerade Medium von Grausamkeit (S. 54). Damit legt Scheuerman einen Widerspruch im „Liberalismus der Furcht“ frei, den dieser kaum mit eigenen Mitteln auflösen könne. Allerdings skizziere Shklar in ihrer späten Auseinandersetzung mit einem typisch US-amerikanischen „Liberalismus der Rechte“"30 ein alternatives Modell des Rechtsstaats, das über den vom „Liberalismus der Furcht" gepriesenen Schutz vor Willkür hinausgeht. Denn innerhalb dieses Modells ,the rule of law itself needed active citizenship to avoid becoming a reified set of rules" (S. 62). Ein richtig verstandener Rechtsstaat benötigt eine kontinuierliche demokratische Auseinandersetzung um die Bedeutung und Reichweite seiner Regeln. Damit stützt Scheuerman die These von einer späten demokratischen Wende in Shklars Denken. Wichtig an seiner Analyse ist die Beobachtung, dass der „Liberalismus der Furcht" zu dieser Hinwendung zum demokratischen Kampf um Rechte nicht in einem Rechtfertigungs- sondern in einem Ergänzungsverhältnis steht. Gerade in

\footnotetext{
27 Gray, John: Agonistic Liberalism, in: Social Philosophy and Policy 12 (1995), H. 1, S. 111-135, hier S. 128.

${ }^{28}$ Siehe zum Beispiel Waldron, Jeremy: Political Political Theory. Essays on Institutions, Harvard UP, Cambridge, MA 2016, S. 30.

${ }^{29}$ Scheuerman, William E.: Law and the Liberalism of Fear, in: Ashenden: Utopia (wie Anm. 6), S. 47-66.

${ }^{30}$ Auch die wichtigsten diesbezüglichen Texte sind in deutscher Sprache nachzulesen in Shklar, Judith N.: Der Liberalismus der Rechte. Hrsg. v. Hannes Bajohr, Matthes \& Seitz, Berlin 2017.
} 
dieser Gestalt ist eine weitere Ausbuchstabierung eines ,agonalen Liberalismus“ mit Shklar lohnenswert.

\section{Neue Texte von Judith Shklar}

Seit 2019 durften sich Shklar-Leser_innen über drei Neuveröffentlichungen freuen. Einerseits legte Hannes Bajohr zwei weitere Bände mit Übersetzungen von ShklarTexten in der handlichen Reihe „Fröhliche Wissenschaft“ des Berliner Verlages Matthes \& Seitz vor. „Über Hannah Arendt“ (ÜHA) dokumentiert Shklars kritische Auseinandersetzung mit der Ikone der politischen Theorie des 20. Jahrhunderts; „Verpflichtung, Loyalität, Exil“ (VLE) gibt einen Einblick in Shklars letztes und leider unvollendet gebliebenes Projekt zu den politischen Verpflichtungen von Exilant_innen. Dieser Einblick wird andererseits durch den Band „On Political Obligation“ (OPO) bereichert, für den Samantha Ashenden und Andreas Hess Texte aus dem Kontext einer Vorlesung an der Harvard University aus dem Jahr 1992 zusammengetragen und editiert haben. Die Chance von Neu-Veröffentlichungen besteht darin, neues Licht auf existierende Deutungskontroversen zu werfen. Dies ist auch hier der Fall. Deswegen konzentriere ich mich auf Aspekte, die für die oben genannten Debatten relevant sind.

\subsection{Ein neuer Blick auf die Romantik und ihre demokratietheoretischen Ressourcen}

Im ersten Teil dieses Rezensionsaufsatzes habe ich argumentiert, dass diejenigen Interpretationen mehr überzeugen, die Shklars liberales Denken weder auf eine minimalistische Variante verkürzen noch ihr einen Mainstream-Universalismus unterstellen, sondern in ihm ein starkes Plädoyer für Demokratie und politisches Handeln sehen. Darüber hinaus hatte sich allerdings die Frage gestellt, ob dies ein kontinuierliches Motiv ihres Denkens ist, oder sich im Spätwerk erst entwickelt; hier schien vieles auf Entwicklungsprozesse hinzudeuten. Die Neuveröffentlichungen können diese These unterstützen, auch weil insbesondere der Band „Über Hannah Arendt“ Texte aus sehr unterschiedlichen Schaffensphasen vereint. Gerade im Vergleich der Texte „Über Hannah Arendt“ und den Vorlesungen aus „On Political Obligation“ wird eine Neubewertung der Romantik deutlich, die, so die hier vertretene These, ein wichtiger Katalysator für die behauptete ,demokratische Wende“ in Shklars Denken ist.

„Über Hannah Arendt“ versammelt ein (gekürztes) Buchkapitel, drei Rezensionen und zwei Nachrufe, in denen sich Shklar mit dem politischen Denken von Hannah Arendt auseinandersetzt. Auch wenn Shklar gerade in den Nachrufen auch genrebedingt viel Wohlwollendes über Arendt zu sagen weiß, bleibt der Ton doch insgesamt ein äußerst kritischer. Dass Shklars „Arendt-Korrekturen“, die Hannes Bajohr in einem anschließenden Essay pointiert rekonstruiert, dem Denken von Hannah Arendt längst „,nicht immer gerecht“ werden (ÜHA, S. 124), hängt viel mit dem scharfen Raster von Shklars Romantik-Kritik aus „After Utopia“ zusammen, durch das sie Arendts Wirken durchweg wahrnahm. Romantisch geprägtes politisches Denken, so 
dokumentiert das die Textsammlung eröffnende Kapitel „Die Romantik der Niederlage“, ersetze den egalitaristischen Individualismus der Aufklärung mit einem antiegalitären, heroischen Individualismus und begründe mit der kulturpessimistischen Analysekategorie der Masse die Vergeblichkeit politischer Gestaltungsoptionen. Das zentrale Problem, das bereits Motive ihres späteren „Liberalismus der Furcht“ andeutet, liegt dabei in der metaphysischen Aufladung des Begriffs des Opfers durch die Erb_innen der Romantik: „Opfersein ist demzufolge unser Schicksal in der natürlichen Welt“ (S. 12f.). Wie Bajohr in seinem Nachwort unterstreicht, blieben ,die wirklichen Opfer [...] dabei aber auf der Strecke, weil sie für den Status des heroischen Einzelnen keine Bedeutung besäßen“ (S. 138). Gerade in ihrer differenzierten Betrachtung der Eichmann-Kontroverse macht Shklar deutlich, dass sie diese problematische Argumentationsfigur auch im Denken von Hannah Arendt am Werk sah (S. 105f.).

Hannah Arendt ist längst nicht die einzige politische Denkerin, die Shklar durch die Brille ihrer Romantik-Kritik wahrnahm. Blickt man in unveröffentlichte Vorlesungsaufzeichnungen, so treffen wir beispielsweise auf den ,romantic poet ${ }^{\star 31}$ Henry David Thoreau, mit dem Shklar zunächst ebenso wenig zimperlich umsprang wie mit Arendt. Thoreaus Parteinahme für die abolitionistische Sache, so hatte Shklar geurteilt, hätten ihm nur zur heroischen Selbstdarstellung gedient; ${ }^{32}$ sein Ruf nach Ungehorsam sei nie von einem bürgerschaftlichen Verantwortungsgefühl getragen gewesen, sondern von dem Wunsch, alle Hindernisse für seine kreative Selbstentfaltung aus dem Weg zu räumen. ${ }^{33}$ Ich greife das Beispiel Thoreau an dieser Stelle heraus, weil an der ihm gewidmeten Vorlesung in „On Political Obligation“ tatsächlich eine Neubewertung der Romantik bei Shklar zu beobachten ist. Und in dieser Neubewertung wird zugleich ein bestimmter Zusammenhang zwischen Shklars ,politischer Skepsis“ (Bajohr) und dem deutlich, was man etwas überspitzt tatsächlich eine „demokratische Wende“ ihres späten Liberalismus nennen könnte.

In der Tat ist das Kapitel zu Thoreau („Civil Disobedience in the 19th Century“) einer der interessantesten Texte aus „On Political Obligation“. ${ }^{34}$ Denn Shklar schreibt Thoreau hier eine Art des politischen Argumentierens zu, die - soweit man dies bei dem work-in-progress-Charakter der Texte sagen kann - ein systematisches Gewicht für ihre eigene Sicht auf die Frage nach politischen Verpflichtungen hat. Denn tatsächlich ist Thoreau für Shklar nicht nur ein politischer Denker des Unge-

\footnotetext{
${ }^{31}$ Shklar, Judith N.: Government 1080 Thoreau, ohne Datum, Papers of Judith N. Shklar, Harvard University Archives HUGFP 118, Box 4, S. 1. Mit freundlicher Genehmigung der Harvard University Archives.

32 Ebd., S. 4.

${ }^{33}$ Shklar, Judith N.: Anarchism, ohne Datum, Papers of Judith N. Shklar, Harvard University Archives HUGFP 118, Box 7. Mit freundlicher Genehmigung der Harvard University Archives.

${ }^{34}$ Die besondere Stellung von Thoreau innerhalb des Buches betonten auch bereits Scheuerman, William E.: Judith N Shklar as theorist of political obligation, in: European Journal of Political Theory 20 (2019), H. 2, S. 366-376; Heins, Volker M.: Thoreau und wir - Judith Shklar über Gewissen, Flucht und Staat. Rezension zu ,On Political Obligation“ und ,Verpflichtung, Loyalität, Exil' von Judith N. Shklar 2019, URL: <https://www.soziopolis.de/lesen/buecher/artikel/thoreau-und-wir-judithshklar-ueber-gewissen-flucht-und-staat/> [Zugriff: 15.11.2019]. Zu Shklars Sicht auf Thoreau, zivilen Ungehorsam und politische Verpflichtung siehe ausführlicher auch Trimcev, Rieke: Judith N. Shklar on Obligation and Disobedience in a ,Society of Strangers“, in: Constellations. An International Journal of Critical and Democratic Theory (im Erscheinen).
} 
horsams, sondern auch der politischen Verpflichtung. So zeichnet Shklar auf engem Raum und mit einer gewissen interpretativen Freiheit nach, wie Praxis und Rechtfertigung zivilen Ungehorsams zu einer Quelle von politischen Bindungen zwischen Mitbürger_innen werden.

Um diesen Gedankengang nachzuvollziehen, muss man zunächst die zentrale Lektion der vorherigen 160 Seiten verstehen, auf denen Shklar Argumente für, vor allem aber auch gegen Verpflichtung und Gehorsam gegenüber Herrscher_innen und Gesetzen von der Antike bis ins Zeitalter der Ideologien nachzeichnet. Knapp zusammengefasst zeigt sich, dass über Regelbefolgen oder Regelbruch immer dann reflektiert wird, wenn sich Individuen in Loyalitätskonflikte verstrickt finden - Shklars Beispiele reichen hier von Sophokles' Antigone über mittelalterliche Reflektionen zum Tyrannenmord bis zu Widerstand und zivilem Ungehorsam im 20. Jahrhundert. ${ }^{35}$ Einer der zentralen begrifflichen Erträge ihrer ideengeschichtlichen Streifzüge ist dabei der bisweilen geradezu mahnende Vorschlag, den Begriff der politischen Verpflichtung von jenem der Loyalität zu trennen. Das falle heute deswegen schwer, weil in Folge des Nationalismus häufig von Loyalitätsbindungen auf politische Verpflichtungen geschlossen werde (OPO, S. 154-156). Während Loyalitäten für Shklar affektive Bindungen an Gruppen sind, seien Verpflichtungen rationale Bindungen an Gesetze (VLE, S. 17). Von dieser Grundlage aus setzt sich Shklar in doppelter Weise von konkurrierenden Perspektiven auf politische Verpflichtungen ab. Gegenüber kommunitaristischen Perspektiven, das macht insbesondere der Text „Das Werk Michael Walzers“ (S. 55ff.) deutlich, weigert sich Shklar konsequent, politische Verpflichtungen aus Gruppenloyalitäten abzuleiten oder auch nur analog zu ihnen zu denken. Anders als die gängigen liberalen Verpflichtungstheorien lehnt es Shklar allerdings ebenso ab, die Rationalität politischer Verpflichtung mit den Mitteln der Theoriearbeit zu ,beweisen'. Vielmehr kommt es ihr darauf an zu verstehen, aus welchen Motiven und welchen affektiven Dynamiken heraus Individuen das Befolgen von Gesetzen als vernünftig verstehen. Nicht Loyalitäten, sondern Loyalitätskonflikte sind für Shklar der Kontext, um nach diesen Motiven und Dynamiken zu fragen - und Thoreau wird hier zu ihrem repräsentativen Beispiel. Thoreau gründet seine Parteinahme für die abolitionistische Sache nicht auf Loyalitäten, da er weder ,den Kameradschaftsgeist des Revolutionärs noch eine mit den Sklaven geteilte Identität" gehabt habe (S. 48). Vielmehr habe sich Thoreau konsequent auf sein Gewissen berufen, das er nicht positiv, sondern negativ, auf dem Weg der skeptischen Rückfrage gerechtfertigt habe: „Warum sollte eine kollektive Stimme mehr Gültigkeit besitzen, einmal davon abgesehen, dass sie mehr Macht auf sich vereint?" (OPO, S. 7). ${ }^{36}$ Thoreau weist das Übergreifen von Loyalitätsansprüchen auf politische Verpflichtungen damit nicht nur zurück, sondern fragt auch nach ungleichen Machtverhältnissen, die hinter geteilten Loyalitäten stehen. Damit ähnelt seine argumentative Strategie der eines ,negativen Egalitarismus“, der

\footnotetext{
35 Einen sehr hilfreichen Überblick über Shklars Harvard-Vorlesungen über politsche Verpflichtungen bietet im Übrigen Hess, Andreas: From Antigone to Martin Luther King. Judith N. Shklar on Moral Reasoning and Disobedience, in: Ashenden: Utopia (wie Anm. 6), S. 239-252.

36 Ich zitiere hier nach der Übersetzung von Hannes Bajohr, dies.: Gewissen und Freiheit, in: Zeitschrift für Politische Theorie 9 (2018), H. 2, S. 167-177, hier S. 171.
} 
nicht so sehr nach den Vorzügen der Gleichheit, sondern nach den gefährlichen politischen Effekten von Ungleichheit fragt. Von zentraler Bedeutung ist darauf aufbauend, dass innerhalb von Shklars sich wandelnden Blick auf Thoreau die Berufung auf das eigene Gewissen nicht länger als selbstzentriert gilt; vielmehr bringe Thoreaus Gewissen ein Freiheitsverständnis zum Ausdruck, das sich konstitutiv auf seine Mitmenschen bezieht. Denn für Shklar steht hinter Thoreaus Weigerung, poll tax zu zahlen, das folgende Argument:

„Unless we identify with the freedom of others, their right to negative freedom, to be allowed all the rights of citizens in a free society, even those who enjoy these rights are limited by being morally constrained, by being implicated in the oppression of others. Within each person, that sets up a moral struggle and a sense of inner oppression" (S. 175).

Indem Thoreau so - wie auch T. H. Green in Großbritannien (S. 137) - die negative Freiheit seiner Mitmenschen zum Maßstab seiner eigenen positiven Freiheit macht, überwinde er die berühmte Dichotomie von Isaiah Berlin. Genau in dieser spezifischen Argumentationsfigur nehme Thoreau eine horizontale Verpflichtung zu seinen Mitmenschen wahr, ohne durch soziale Näheverhältnisse, ohne durch Loyalitäten an sie gebunden zu sein.

Für die Möglichkeit, den „Liberalismus der Furcht“ mit demokratischen Prinzipien in ein engeres Verhältnis zu setzen als das einer Zweckehe, sind diese Thoreau'sche Lektion und die mit ihr verbundene Neubewertung der Romantik eine wichtige Weichenstellung. Shklars Liberalismus war in seiner Rezeptivität für jene Stimmen, die normalerweise ausgeschlossen und stumm sind, immer zutiefst individualistisch. Doch wie waren Individualismus und negativer Egalitarismus miteinander zu vereinbaren? Der Individualismus der radikalen Aufklärung war ein zwar egalitärer Individualismus, doch er erkaufte sich seinen Egalitarismus durch einen Rationalismus, das heißt, er sah die Gleichheit in der geteilten Vernunft aller Individuen verbürgt. ${ }^{37}$ Diesen Rationalismus wollte Shklar, die Menschen als fühlende Lebewesen sah, nicht teilen. Der Individualismus der Romantik dagegen, der das Einzigartige und das mögliche Genie im Menschen suchte, schien Shklar lange Zeit als anti-egalitär und elitistisch. Genau diese Zweifel widerlegte die ThoreauLesart der späten Judith Shklar.

\subsection{Wie radikal ist Shklars Denken wirklich?}

Bleibt die Frage, wie „radikal“ und „aktivistisch“ der späte und demokratisierte „Liberalismus der Furcht" Judith Shklars wirklich ist, und wie weit die Bezeichnung als ,agonale“ politische Theorie tatsächlich trägt. Im ersten Teil dieses Aufsatzes hatte ich Giunia Gattas Bezeichnung von Shklars Ansatz als einen agonalen durchaus viel abgewinnen können; zugleich hatte ich aber gefragt, ob es nicht gewinnbringender sein könnte, sie tatsächlich als liberale agonale Theoretikerin zu lesen, anstatt Shklar zu einer „radikalen“ und ,aktivistischen“ Theoretikerin zu stilisieren.

37 Zum Vergleich von aufklärerischem und romantischem Individualismus siehe Shklar: After Utopia (wie Anm. 9), S. 82-84. 
Dass Thoreau eine solche Aufwertung und seine Verteidigung des Ungehorsams eine solche repräsentative Stellung erhält, scheint auf den ersten Blick ein Indiz dafür, dass Shklar tatsächlich ,radikaler' wird. Hier möchte ich allerdings drei Einwände anbringen. Der erste Einwand stützt sich auf die Art und Weise, wie Shklar die Einsichten ihrer Harvard-Vorlesungen in ,Verpflichtung, Loyalität, Exil“ weiterentwickelte; der zweite und der dritte Einwand schließlich haben mit zwei blinden Flecken zu tun, die sich aus Shklars Überlegungen zum demokratischen Handeln speisen.

Trotz der zentralen Stellung von Thoreau in „On Political Obligation“ sollte nicht vergessen werden, dass hinter Shklars Reflexionen über Widerstand und Ungehorsam stets die Frage nach politischen Verpflichtungen stand - also die Frage, warum und unter welchen Bedingungen Menschen den Gesetzen des Landes, in dem sie leben, Folge leisten und leisten sollen. In der Vorlesung „Consent and Obligation“ setzt sich Shklar zum ersten Mal explizit mit den damals gängigen liberalen Verpflichtungstheorien auseinander. In „On Political Obligation“ wird diese Vorlesung durch einige Tafelnotizen repräsentiert, die Themen und Struktur der Vorlesung anzeigen, aber Shklars Argumentation nicht im Einzelnen abbilden. Tatsächlich ist das auch von den Herausgebenden beklagte Fehlen eines ausformulierten Manuskripts für diese Vorlesung äußerst bedauernswert. Denn es wird zwar deutlich, dass Shklar der Zustimmungs-, der fair-play- und der Dankbarkeitstheorie politischer Verpflichtung kritisch gegenüberstand, doch es wird nicht deutlich, wie grundsätzlich diese Kritik ausfällt. Für die Einschätzung der argumentativen Reichweite ihrer zu Thoreau angestellten Überlegungen ist dies aber entscheidend.

Wie Samantha Ashenden und Andreas Hess in ihrer „Note on Sources“ (S. 233) anführen, gibt es zusätzlich zu den Tafelnotizen zu dieser Vorlesung in Shklars Nachlass noch ein Manuskript mit dem Titel „MrCon2“, das allerdings aus sehr fragmentarischen, stichpunktartigen Notizen besteht. ${ }^{38}$ Sicherlich gehörte die Frage, ob diese in das Buch zu übernehmen seien oder nicht, zu den schwierigeren Fragen dieses an schwierigen Fragen nicht armen Editionsprojektes. So finden sich in Shklars Nachlass zu vielen der einzelnen Vorlesungen mehrere Manuskripte oder Notizen. Ashenden und Hess haben zudem viele wertvolle Materialien und Informationen von einem ehemaligen research assistant von Shklar erhalten und aufbereitet. Es galt stets abzuwägen, welche Texte in das Buch eingehen würden, und im Ergebnis ist aus vielen sehr nachvollziehbaren Einzelentscheidungen ein zugleich gut lesbarer und sehr vollständiger Text geworden. Einzig allein im Falle der Vorlesung „Consent and Obligation“ wäre zu fragen, ob den Leser_innen nicht ein sehr wichtiger Aspekt von Shklars Reflektionen über politische Verpflichtungen vorenthalten bleibt. Tatsächlich sind die Formulierungen in „MrCon2“ längst nicht immer eindeutig und lassen viele Fragen offen, was sicher allein schon aus Rücksicht auf eine Autorin, die ihre Vorlesungsnotizen für die Lehre, und nicht mit Veröffentlichungsabsicht schrieb (S. 216), gegen einen Abdruck spricht. Allerdings wurden für die Vorlesungen zu John Locke, David Hume, Jean-Jacques Rousseau und Imma-

\footnotetext{
38 Shklar, Judith N.: MrCon2, ohne Datum, Papers of Judith N. Shklar, Harvard University Archives HUGFP 118, Box 7. Mit freundlicher Genehmigung der Harvard University Archives.
} 
nuel Kant ebenfalls Texte in einem ähnlich unfertigen und stichpunktartigen Duktus abgedruckt (S. 223, S. 108-120).

Was hätte man in „MrCon2“ für die Frage nach der Radikalität von Shklars Blick auf Ungehorsam und Verpflichtung finden können? Shklar stellt zunächst all diejenigen Ansätze, die sich als Alternativen zur klassischen Zustimmungstheorie politischer Verpflichtung ausgeben, als heimliche Zustimmungstheorien heraus. Auch die Prinzipien von fair play und Dankbarkeit begründeten im Grunde nur die hypothetische Zustimmung von rationalen Individuen zu gerechten Gesetzen. ${ }^{39}$ Allerdings, so wendet Shklar ein, werde damit immer nur die Zustimmung zu einer idealisierten liberalen Demokratie begründet, und nie die Verpflichtung zu realen liberalen Demokratien plausibel gemacht, in denen wir in einer „Gesellschaft von Fremden“ zusammenleben würden. ${ }^{40}$ Für diese schlägt Shklar dann einen recht abgeklärten, und weniger anspruchsvollen Grund vor, den Gesetzen Folge zu leisten: Das Recht setze Normen, um das Handeln unter Fremden zu koordinieren; es informiere uns über Sanktionen über die sozialen Normen der Gesellschaft; und es sei Teil eines größeren rechtstaatlichen Systems. ${ }^{41}$ Shklar räumt in dieser Vorlesung ein, dass es zudem ,terrible moments“ gebe, in denen ziviler Ungehorsam gerechtfertigt sei. ${ }^{42}$ Dennoch fallen politische Verpflichtungen hier weitaus grundsätzlicher aus, als es eine „radikale“, grundsätzlich herrschaftskritische Perspektive erlauben würde. Man wird sich zudem fragen, ob Shklar nicht letztendlich auf die Einsicht der Bürger_innen setzt - also genau mit jener Verkürzung von Menschen auf ihre Rationalität arbeitet, für die sie andere Verpflichtungstheorien kurz zuvor kritisiert hatte.

Zieht man allerdings „Verpflichtung, Loyalität, Exil“ hinzu, wird ein Unterschied deutlich. Denn Shklar will mitnichten argumentieren, dass Verpflichtungen nicht rational wären - Rationalität gehört für sie zum Kern von politischen Verpflichtungen. Vielmehr erwartet sie von einer Verpflichtungstheorie, (a) eine Form von Rationalität zu bestimmen, die Individuen tatsächlich erreichen können, und (b) auch verständlich zu machen, wie diese realistische Form von Rationalität entsteht. Und an dieser Stelle führt die These, dass wir in einer ,Gesellschaft von Fremden ' leben, mitten hinein in die Überlegungen aus ,Verpflichtung, Loyalität, Exil““. Hier schaut Shklar von einem besonderen Typus von Fremden aus auf politische Verpflichtungen: Sie nimmt die Perspektive von Exilant_innen ein. Exilant_innen finden sich besonders häufig und besonders deutlich jenen Loyalitätskonflikten ausgesetzt, die, so könnte man vermuten, auch für pluralistische ,Gesellschaften von Fremden ' typisch sind. Shklar merkt explizit an, dass viele ihrer Überlegungen über tatsächliche Exilant_innen auch für Menschen gelten, denen ein ,inneres Exil“ droht für Menschen also, die sich aufgrund der sie umgebenden Ungerechtigkeiten von allen Gruppenbindungen lossagen (VLE, S. 50). Hier findet sich dann der Bogen zu Thoreau zurückgeschlagen - denn auch Thoreau bezeichnete Shklar als inneren Exilanten (OPO, S. 174). Möglicherweise wird Shklars oben referierte pragmatische

\footnotetext{
39 Ebd., S. 7.

40 Ebd, S. 4.

41 Ebd., S. 8ff.

42 Ebd., S. 11.
} 
Verteidigung von politischen Verpflichtungen plausibel, wenn man sie als jene Form von Bindung an das Gesetz versteht, die Fremde unter Fremden gerade deswegen als vernünftig einsehen können, weil sie um die oftmals unentscheidbare Natur und die vielen Risiken von Verrat und Ungerechtigkeit, die Loyalitätskonflikte mit sich bringen, besonders wissen. Dies wäre dann in der Tat ein Zugang zu politischen Verpflichtungen, der liberal bleibt, dabei aber wichtige Punkte der kommunitaristischen und der radikaldemokratischen Kritik am Liberalismus aufnehmen kann.

Wenn Shklar im Zuge ihrer Thoreau-Interpretation zugleich auch widerständiges politisches Handeln aufwertet, sollte dabei nicht aus den Augen verloren werden, dass es sich dabei innerhalb ihrer Reflexionen um ein einsames und advokatorisches Handeln handelt. Ein ,Wir' ist in dieser Art des Handelns gleich im doppelten Sinne verwehrt. Weder verbindet Thoreau mit denen, deren Ungleichbehandlung er kritisiert, irgendeine Form von Solidarität, noch handelt er in einer organisierten Gruppe von Gleichgesinnten. Noch drängender scheint aber die Frage, ob diese Figur des sich einsam auf das eigene Gewissen berufenden Opponenten tatsächlich so verallgemeinerungsfähig ist, wie es Shklar vorzuschweben schien. In dieser Hinsicht ist der Beitrag von Katrina Forrester in „Between Utopia and Realism“ erhellend. Forrester zeichnet in ihrem Aufsatz „Experience, Ideology, and the Politics of Psychology“ nach, wie psychologische Fragestellungen für Shklar zentral werden. ${ }^{43}$ Shklar, so Forrester, habe in den 1960er Jahren einen alternativen Pfad der Ideologiekritik erschlossen, ,one that judged an ideology by unmasking not only whose interests it served but also whose experiences it failed to capture" (Forrester in Ashenden: Utopia, S. 143). Diesen Ausgangspunkt habe Shklar zunehmend zur Annahme einer primären, von Gruppenideologien unbehelligten Erfahrung des Individuums geführt, der sie unter Anleitung von Rousseau als ,moral psychology“ Rechnung tragen wollte (S. 144). Für Forrester führt diese Gegenüberstellung von Ideologie und Erfahrung in die sowohl theoretische als auch politische Sackgasse eines „radikalen Individualismus“ (S. 153). Man muss Forrester angesichts der oben referierten Positionen von Shklar nicht unbedingt zustimmen, dass Shklar die Frage von politischer Verpflichtung subjektivistisch auf individuelle Gewissensfragen verkürze und damit eine Flucht vor institutionellen Überlegungen antrete (S. 151). Tatsächlich stellt sich aber die Frage, ob die affektive Bindung an das ,Wir' einer Gruppe nicht zumindest für die Ermöglichung der Artikulation der Leidenserfahrungen von Minderheiten, die von ideologischen Denkgewohnheiten unsichtbar und unsagbar gehalten werden, wichtig sind. Loyalitäten sind insofern schützenswert für Shklar, als dass sie der politischen Verfügung entzogen sein sollten. Da sie affektiver Natur sind und oftmals nicht willentlich abgelegt werden können, sollten Menschen nicht durch „Loyalitätsbeweise“ zum Staat dazu gezwungen werden, ihnen abzuschwören. Dies, so Shklar, sei der „Pfad der Ungerechtigkeit“ (VLE, S. 53). Shklar scheint allerdings stets davon auszugehen, dass jene Loyalitäten, über die das Gewissen hinausgehen kann, immer schon existieren. Wenn aber Loyalitäten eine Ermöglichungsbedingung der Artikulation von Leidenserfahrungen sind, müss-

43 Forrester, Katrina: Experience, Ideology, and the Politcs of Psychology, in: Ashenden: Utopia (wie Anm. 6), S. 136-157. 
te Shklar ihnen eventuell noch eine stärkere Rolle einräumen. ${ }^{44}$ Dann aber würde sich die Frage stellen: Wie lange verdienen Loyalitätsbindungen aus der Sicht eines „Liberalismus der Furcht“ unser ermutigendes Wohlwollen, und ab wann verdienen sie die Skepsis, mit denen Shklar ihnen sowohl in ihren Texten als oft genug auch im wirklichen Leben begegnete?

Akzeptiert man dennoch Shklars Ausgangspunkt, bleibt immer noch die Frage nach den Möglichkeiten und Grenzen advokatorischen Handelns. Denn advokatorisches Handeln impliziert immer, dass Ungleichheit und die aus ihr erwachsenden Ungerechtigkeiten repräsentiert werden können. Dass die psychische Realität des Leidens niemals unmittelbar transparent und intelligibel wird, war Shklar vollends bewusst. Gerade weil sie sich nicht objektiv beschreiben lässt, behandelte Shklar sie in vielen Texten als blackbox, deren Inhalt allerdings indirekt, durch Geschichten unterschiedlicher hochkultureller und vernakularer Genres, in die Theoriearbeit mit einbezogen wird. Gleich zwei Beiträge gehen in „Between Utopia and Realism“ dieser narrativ-poetischen Seite von Shklars Werk nach. So betont Tracy B. Strong, dass die Einwebung literarischen Materials ,forces the reader to acknowledge what her or she only imperfectly knows". ${ }^{45}$ James Brown und Thomas Osborne schlagen darüber hinaus vor, in Shklars Bemühen, über den Rückgriff auf literarische Materialien eine Rationalisierung dessen zu vermeiden, was in der Politik irrational ist und bleibt, ein Zugeständnis an die Romantik zu sehen; ${ }^{46}$ sie zeigen damit eine andere Ebene einer Neubewertung der Romantik bei Shklar an, die ich oben behauptet habe.

Während Shklar das Problem der Repräsentation von Leidenserfahrungen für ihre schriftstellerische Praxis sehr bewusst war, findet sich für die politikpraktische Ebene keine vergleichbare Reflexion. Die „Krise der Repräsentation“ traf Shklar offenbar nie so tief, als dass sie sich der Frage gestellt hätte, zu welchen neuen Ungerechtigkeiten die Repräsentation von Ungerechtigkeit und Grausamkeit führt. Jean-François Lyotard, ein anderer bedeutender Theoretiker des 20. Jahrhundert, der den Begriff des Opfers zu einer zentralen Kategorie seiner politischen Theorie machte, ging davon aus, dass Opfer niemals repräsentiert, sondern höchstens „,bezeugt“" werden könnten..$^{47}$ Er unterzog die Übernahme der Stimmen der anderen einer grundlegenden Problematisierung, für die sich bei Shklar keine Entsprechung findet. Sie hielt advokatorisches Handeln für grundsätzlich möglich; es scheint fast auch primär der Typus demokratischen Handelns gewesen zu sein, der ihr seit „Über Ungerechtigkeit" vorschwebte.

\footnotetext{
44 Andeutungen dazu ließen sich in Shklars Überlegungen zu den unintendiert positiven Effekten finden, was sie den ,indirekten Wert“ von „,sekundärem Snobismus“ nennt: Gerade in einer ,ausschließenden Gruppe gleichgesinnter Menschen“ würden wir „Gleichheit, Intimität und Brüderlichkeit“ erlernen, siehe Shklar: Laster (wie Anm. 5), S. 154f.

45 Strong, Tracy B.: Literature and the Imagination, in: Ashenden: Utopia (wie Anm. 6), S. 101-115, hier S. 102.

46 Brown, James/Osborne, Thomas: Imaginative Literature and Political Theory: An Engagement, in: Ashenden: Utopia (wie Anm. 6), S. 116-135, hier S. 117. Ein ähnliches Argument, wenn auch weniger differenziert, unterbreitet in dieser Hinsicht Gatta: Liberalism (wie Anm. 4), S. 56.

47 Lyotard, Jean-François: Le Différend, Éditions de Minuit, Paris 1983.
} 
Alle drei Punkte sind meiner Auffassung nach ein Indiz dafür, dass Shklars politische Theorie in manchen Gesichtspunkten bestimmte, typischerweise von Radikaldemokrat_innen aufgeworfene Probleme bearbeiten kann. Gleichzeitig wird aber deutlich, dass ihre politische Theorie selbst nicht als ,radikal' bezeichnet werden kann. Mit den blinden Flecken sind zwei Punkte benannt, die einer Bearbeitung bedürfen; es scheint aber nicht ausgeschlossen, dass sie bearbeitbar sind.

\section{Schluss}

Die zunehmende Zahl an Veröffentlichungen zeigt, dass das politische Denken von Judith Shklar zunehmend zu einer Projektionsfläche für die unterschiedlichsten politiktheoretischen und zuweilen auch politischen Hoffnungen wird. Mit den Erwartungen wächst aber auch das Enttäuschungspotential. Hier und da macht sich auch unter Autor_innen der ersten Stunde des Shklar-Hypes der letzten Dekade eine beginnende Ernüchterung bemerkbar. ${ }^{48}$ Zunehmend wird auch überrascht festgestellt, dass Shklars Vision sich trotz aller Absatzbewegungen in institutioneller Hinsicht doch kaum von derjenigen politischen Ordnung unterscheidet, die ihr Freund und Kollege John Rawls fulminant gerechtfertigt hatte. ${ }^{49}$ Also doch liberaler Mainstream? Noch schlimmer, bietet der „Liberalismus der Furcht“ nur jenen Freund_innen der liberalen Demokratie, die sich selbst für kritischer halten als ihre status-quo-verliebten Mit-Liberalen, eine etwas plausiblere Rechtfertigung der bestehenden Ordnung an? Zu diesem Urteil sah sich jüngst Veith Selk auf der Basis des Shklar-Aufsatzes über den „Liberalismus der Furcht“" veranlasst. ${ }^{50}$

Es lohnt sich an dieser Stelle, die Erwartungen zu rekalibrieren und zu hinterfragen, was denn ein ,,anderer Liberalismus“ (Müller) „für das 21. Jahrhundert“ (Gatta) überhaupt leisten können soll. Grundlegend andere institutionelle Ordnungsentwürfe wird man dabei von einer Theoretikerin, die sich zeitlebens entschieden als Liberale begriff, kaum erwarten wollen. Auch habe ich in diesem Beitrag die Auffassung vertreten, dass Shklars politische Theorie nicht selbst „radikal“ oder ,aktivistisch“ ist. Allerdings konnten neue Texte über und von Shklar zeigen, dass sie in ihren späten und zum Teil leider unvollendet gebliebenen Schriften erstens die Bedeutung von demokratischem Handeln für liberale Institutionen aufwertet, und dabei in Hinsicht auf politische Verpflichtungen zweitens wichtige Kritikpunkte an gängigen liberalen Ansätzen aufnehmen kann. Auch wenn Shklar dabei weder ein politiktheoretisches System ausarbeitet, noch in politische Rezepte übersetzt, ist damit für einen ,,anderen Liberalismus" schon viel gewonnen.

\footnotetext{
48 So zum Beispiel bei Forrester: Experience (wie Anm. 48).

49 Fives: Monism (wie Anm. 17).

50 Selk: Veralten (wie Anm. 3).
} 


\section{Besprochene Literatur}

- Ashenden, Samantha/Hess, Andreas (Hrsg.): Between Utopia and Realism. The Political Thought of Judith N. Shklar, 304 S., University of Pennsylvania Press, Philadelphia/PA 2019.

- Gatta, Giunia: Rethinking Liberalism for the 21st Century. The Skeptical Radicalism of Judith Shklar, 164 S., Routledge, New York/NY u.a. 2018.

- Müller, Jan-Werner: Furcht und Freiheit. Für einen anderen Liberalismus, 171 S., Suhrkamp, Berlin 2019.

- Shklar, Judith N.: On Political Obligation, 264 S., Yale UP, New Haven/CT u.a. 2019.

- Shklar, Judith N.: Verpflichtung, Loyalität, Exil. Mit einem Essay über Michael Walzer, 88 S., Herausgegeben und übersetzt von Hannes Bajohr, Matthes \& Seitz, Berlin 2019.

- Shklar, Judith N.: Über Hannah Arendt. Herausgegeben und mit einem Nachwort versehen von Hannes Bajohr, 120 S., Matthes \& Seitz, Berlin 2020.

Funding Open Access funding enabled and organized by Projekt DEAL.

Open Access Dieser Artikel wird unter der Creative Commons Namensnennung 4.0 International Lizenz veröffentlicht, welche die Nutzung, Vervielfältigung, Bearbeitung, Verbreitung und Wiedergabe in jeglichem Medium und Format erlaubt, sofern Sie den/die ursprünglichen Autor(en) und die Quelle ordnungsgemäß nennen, einen Link zur Creative Commons Lizenz beifügen und angeben, ob Änderungen vorgenommen wurden.

Die in diesem Artikel enthaltenen Bilder und sonstiges Drittmaterial unterliegen ebenfalls der genannten Creative Commons Lizenz, sofern sich aus der Abbildungslegende nichts anderes ergibt. Sofern das betreffende Material nicht unter der genannten Creative Commons Lizenz steht und die betreffende Handlung nicht nach gesetzlichen Vorschriften erlaubt ist, ist für die oben aufgeführten Weiterverwendungen des Materials die Einwilligung des jeweiligen Rechteinhabers einzuholen.

Weitere Details zur Lizenz entnehmen Sie bitte der Lizenzinformation auf http://creativecommons.org/ licenses/by/4.0/deed.de.

Rieke Trimcev ist Nachwuchsgruppenleiterin am Arbeitsbereich für Politische Theorie und Ideengeschichte der Universität Greifswald. Sie studierte in Hamburg und Paris Politikwissenschaft, Romanistik und Erziehungswissenschaft und wurde 2017 an der Universität Augsburg mit einer Arbeit über politische Metaphern promoviert. Arbeitsschwerpunkte sind Begriffs- und Metapherngeschichte, agonale Demokratietheorien, Theorien politischer Verpflichtung sowie Erinnerungsforschung. 